\title{
Poluição ambiental, residência materna e baixo peso ao nascer
}

\author{
Environmental pollution, maternal residence and low birth weight \\ Contaminación ambiental, residencia materna y bajo peso al nacer
}

\author{
Marli Terezinha Stein Backes', Maria Cristina Flores Soares" \\ ' Universidade Federal de Santa Catarina (UFSC), Programa de Pós-Graduação em Enfermagem. \\ Florianópolis-SC, Brasil. \\ "Fundação Universidade Federal do Rio Grande (FURG), Departamento de Ciências Fisiológicas. \\ Rio Grande-RS, Brasil.
}

Submissão: 23/09/2009 Revisão: 20/11/2010 Aprovação: 11/01/2011

\section{RESUMO}

Estudo caso-controle, que objetivou analisar os fatores de risco associados ao baixo peso ao nascer de recém-nascidos de mães de Rio Grande-RS residentes nas proximidades da área industrial. Foram entrevistadas mães que deram à luz nas maternidades do município, durante os meses de abril a novembro de 2003. A amostra compreendeu 138 casos e 409 controles. Foi realizada análise estatística bivariada e multivariada. O Baixo Peso ao Nascer (BPN) manteve-se associado positivamente com natimortos prévios, BPN prévios, presença de hipertensão arterial durante a gestação e ameaça de aborto durante a gravidez atual. Foi possível identificar os principais fatores de risco a que estão expostas as gestantes e que interferem no peso ao nascer de seus filhos, os quais vêm somar-se àqueles decorrentes de uma maior exposição a poluentes, por residirem próximo às indústrias.

Descritores: Estudos de casos e controles; Fatores de risco; Habitação; Poluição ambiental; Recém-nascido de baixo peso.

\section{ABSTRACT}

Case-control study, which aimed to analyze the risk factors associated with low birth weight of newborns of mothers from Rio Grande-RS that resided in the proximities of the industrial area. Mothers that gave birth in the maternities of the municipality were interviewed during the months of April to November of 2003 . The sample comprehended 138 cases and 409 controls. It was performed the bivariate and multivariate statistical analysis. The Low Birth Weight (LBW) maintained itself positively associated with previous stillborns, previous LBWs, the presence of arterial hypertension during the pregnancy, and the threat of abortion during the current pregnancy. It was possible to identify the main factors of risk to which pregnant women are exposed and that interfere with birth weight of their children, which are in addition to those resulting from a greater exposure to pollutants, because they live near the industries.

Key words: Case-Control Studies; Risk Factors; Housing; Environmental pollution; Infant Low Birth Weight.

\section{RESUMEN}

Estudio caso-control, que tuvo como objetivo analizar los factores de riesgo asociados con el bajo peso al nacer de los recién nacidos de madres de Rio Grande- RS, residentes cerca de la zona industrial. Estudio caso-control, que tuvo como objetivo analizar los factores de riesgo asociados con el bajo peso al nacer de los recién nacidos de madres de Rio Grande- RS, residentes cerca de la zona industrial. Se entrevistaron a las madres que dieron a luz en maternidades del municipio, durante los meses de abril a noviembre de 2003. La muestra incluyó 138 casos y 409 controles. Se realizó una análisis estadística bivariada y multivariada. El bajo peso al nacer (BPN) se mantuvo asociado positivamente con la muerte del feto, bajo peso al nacer previos, presencia de hipertensión durante el embarazo y con la amenaza de aborto durante el embarazo actual. Fue posible identificar los principales factores de riesgo a que las mujeres embarazadas están expuestas y que interfieren con el peso al nacer de sus hijos, que se suman aquellos que resultan de una mayor exposición a los contaminantes, debido a que viven cerca de las industrias.

Palabras clave: Estudio de casos y controles; Factores de riesgo; Habitación; Contaminación ambiental; Bajo peso al nacer. 


\section{INTRODUÇÃO}

Vários estudos têm demonstrado os efeitos da poluição ambiental sobre a saúde das pessoas, nos diferentes períodos da vida $^{(1-3)}$. No entanto, em algumas fases os danos causados à saúde pela poluição podem ser mais dramáticos e irreversíveis, decorrentes de processos mutagênicos, teratogênicos e carcinogênicos, a médio e longo prazo, de caráter cumulativo e combinado. Isso é especialmente verdadeiro quando nos referimos à saúde do binômio materno-infanti $i^{[1,2)}$. Nesse caso, os agravos geralmente são devidos a uma exposição direta a determinados fatores de risco, como no caso da exposição de mulheres que trabalham em ambientes insalubres ou, indiretamente, quando estas residem em áreas potencialmente de risco. Estudos têm mostrado que fatores ambientais, como a poluição atmosférica e das águas, podem levar a agravos de saúde, interferindo na reprodução, nas condições gestacionais, nas condições de vida do concepto, assim como podem também contribuir para o baixo peso ao nascimento ${ }^{(2,4)}$.

O Baixo Peso ao Nascer (BPN) é definido pela Organização Mundial da Saúde (OMS) como o peso de nascimento inferior a $2.500 \mathrm{~g}$, sendo considerado um fator importante, pois contribui para o aumento da morbimortalidade infantil, principalmente entre os recém-nascidos ${ }^{(5)}$, como também no primeiro ano de vida, uma vez que essas crianças apresentam risco de morrer quinze vezes maior, quando comparadas com aquelas que nascem com peso adequado ${ }^{(6)}$.

O peso ao nascer é também um indicador geral, utilizado para avaliar os níveis de saúde de uma população, por estar associado às condições socioeconômicas do país ao qual ela pertence ${ }^{(5)}$. Assim, as maiores prevalências de BPN (em torno de $90 \%$ ) são encontradas nos países em desenvolvimento, que apresentam piores condições de vida ${ }^{(7)}$. Porém, o peso ao nascer passou a ser um problema de saúde pública mesmo nos países desenvolvidos, devido a situações ambientais desfavoráveis ${ }^{(8)}$.

Acredita-se que as causas da ocorrência de BPN sejam multifatoriais ${ }^{(9)}$, sendo que os diversos fatores de risco que contribuem para o BPN são: o retardo do crescimento intra-uterino, a prematuridade, peso materno pré-gestacional menor que $50 \mathrm{~kg}$, intervalo interpartal menor que dezoito meses, desnutrição materna, história de partos prematuros, filho(os) anterior(es) com BPN, primigestas, gestantes multíparas com mais de três filhos, hábito de fumar e fumo passivo, pouca escolaridade materna, mães adolescentes, idade materna acima de 35 anos, mães que não vivem com marido ou companheiro (mulheres não unidas), ausência e/ou insuficiência de cuidados pré-natais, cesarianas, entre outros, os quais podem agir de forma isolada ou associadamente ${ }^{(5,9-14)}$.

No que se refere ao município de Rio Grande-RS, estudo, realizado no período de janeiro a junho de 2003, constatou prevalência de BPN de 10,5\%, quando foram avaliados todos os nascimentos ocorridos no município ${ }^{(15)}$. Para conhecer o impacto da poluição ambiental nesse município sobre esse indicador, especialmente sobre a parcela da população que reside nas proximidades das áreas industriais, buscou-se investigar se as mulheres que residem nas comunidades próximas ao parque industrial do município de Rio Grande-RS, possivelmente mais sujeitas à exposição de poluentes ambientais provenientes das indústrias de fertilizantes e do refino de petróleo, sofrem influência desse fator durante a gestação, interferindo nas condições de saúde do concepto ao nascimento. Assim, o estudo buscou analisar os fatores de risco associados ao baixo peso ao nascer de recém-nascidos de mães residentes nas comunidades próximas ao parque industrial do município de Rio Grande-RS.

\section{MÉTODO}

Estudo caso-controle, realizado no município de Rio Grande-RS, no período de abril a novembro de 2003. Os critérios de inclusão dos casos foram assim definidos: recém-nascidos de partos únicos, vivos ou mortos, filhos de mães residentes no município, que deram à luz nas maternidades dos dois hospitais gerais do município, durante o período do estudo, com BPN, ou seja, pesando menos de 2.500 gramas. Foram considerados controles as três crianças, nas mesmas condições, que nasceram imediatamente após o caso, no mesmo hospital, com peso ao nascer igual ou superior a 2.500 gramas.

O tamanho da amostra foi definido para alcançar um nível de confiança de $95 \%$, um poder de $80 \%$ e um risco relativo de 2,50, estando a prevalência de exposição entre os controles em $9 \%$. Estimou-se que, trabalhando com uma proporção de três controles para cada caso, e incluindo $10 \%$ para perdas e $15 \%$ para controle das variáveis de confusão, seriam suficientes 139 casos (recém-nascidos com BPN) e 417 controles (recém-nascidos com peso normal).

Foi aplicado um questionário a 547 mães, ainda durante a internação hospitalar na maternidade, por um entrevistador treinado, tendo sido consideradas para o estudo apenas mães residentes no município de Rio Grande-RS. Dessa maneira, a partir dos critérios de inclusão, foram consideradas todas as mães dos casos e controles do município e, as mesmas foram classificadas como expostas ou não expostas, tanto em ralação aos casos como em relação aos controles. Assim, tanto para os casos como para os controles, as mães poderiam estar residindo nas áreas próximas às indústrias de fertilizante e do refino de petróleo ou não.

Foram consideradas expostas as mães que residiam nas proximidades da área industrial do município de Rio Grande-RS, por mais de nove meses, levando em conta que o tempo de gestação envolve nove meses. A fim de delimitar a área do estudo, foram incluídos os bairros Lar Gaúcho, Navegantes, parte do bairro Centro, parte do Getúlio Vargas (BGV), Santa Tereza, Vila Xavier, Vila Santo Antônio e, ainda, a Vila da Mangueira, que fica no caminho entre a refinaria de petróleo e as indústrias de fertilizantes. Todos esses bairros apresentam proximidade semelhante em relação à refinaria de petróleo e indústrias de fertilizantes, conforme mostra o Mapa da Figura 1 a seguir, referente à cidade de Rio Grande-RS.

As variáveis relacionadas aos fatores ambientais avaliadas nessa pesquisa foram: o local de residência materna (se exposto ou não exposto), o tempo de residência da mãe na área exposta, (considerando esse tempo da seguinte maneira: de 


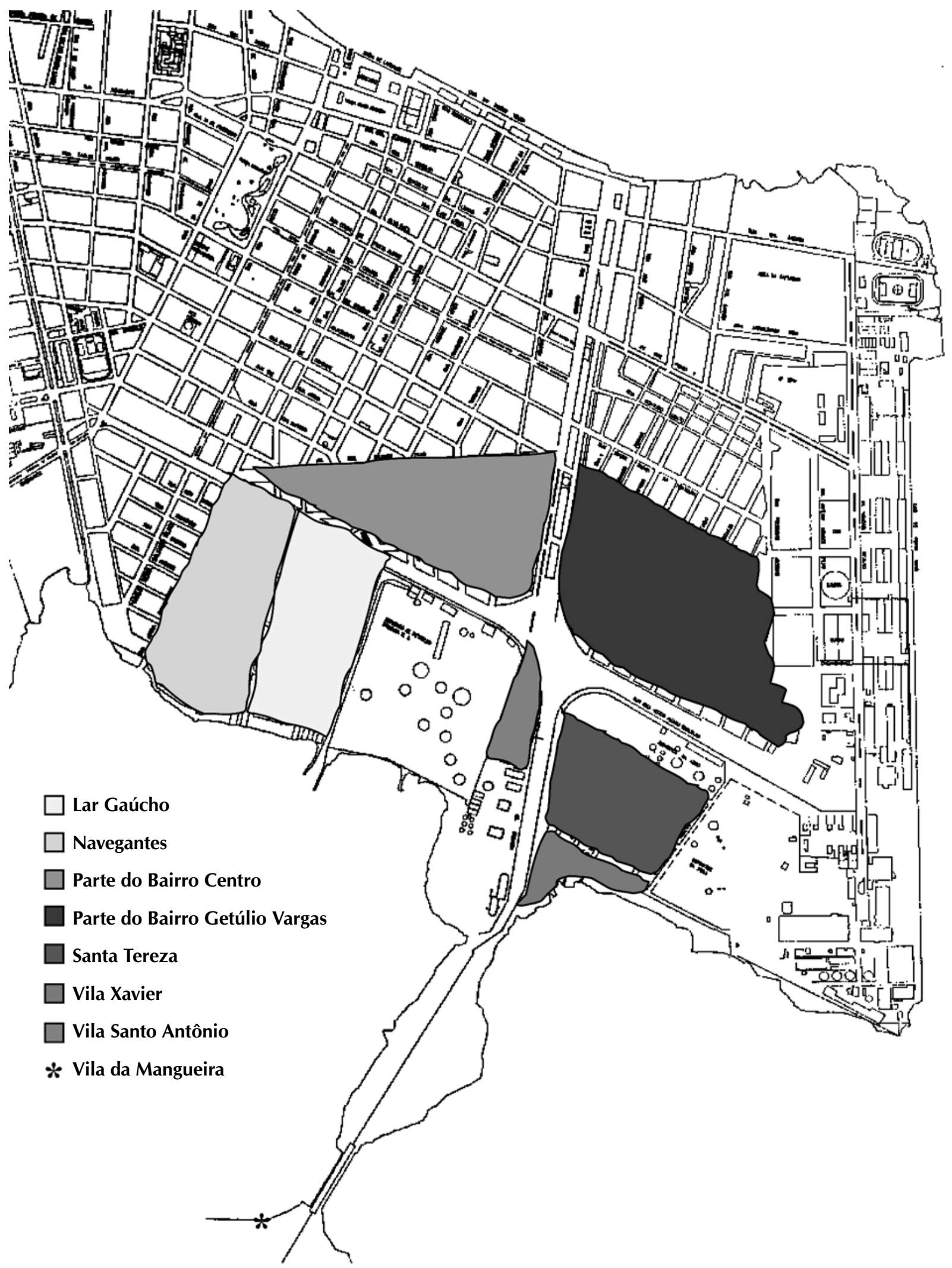

Figura 1: Área do estudo 
zero a nove meses, de 10 meses a cinco anos e, mais de cinco anos), o local de residência anterior da mãe (se exposto ou não exposto) e o local de trabalho da mãe durante a gestação (se exposto ou não exposto). Além dessas variáveis, também foram incluídas nesse estudo variáveis socioeconômicas e demográficas, condições de moradia, características biológicas maternas, fatores maternos reprodutivos, o fumo materno e o fumo passivo, intercorrências durante a gestação e assistência pré-natal e o tipo de parto.

Os dados coletados foram digitados duas vezes, por pessoas diferentes, com o auxílio de microcomputador, através do programa Epi Info 6.04 versão 2001. Posteriormente, foi realizada a limpeza dos dados, corrigindo erros de amplitude e de consistência. Os dados foram analisados, usando o programa SPSS 8.0 para realizar a análise bivariada e multivariada.

Os dados foram avaliados pelo método de regressão logística não condicional, obtendo-se as razões de Odds e intervalos de confiança em todas as análises, utilizando-se um nível de significância de 0,05. Na análise bivariada, o efeito de cada uma das variáveis foi analisado isoladamente. Na regressão logística multivariada foi efetuado o ajuste para todas as variáveis que permaneceram no modelo hierárquico previamente estabelecido, ou seja, que apresentaram um nível de significância menor que 0,20.

Em relação aos aspectos éticos respeitou-se a Resolução $n^{\circ}$ 196/96 ${ }^{(16)}$ do Conselho Nacional de Saúde / Ministério da Saúde, sobre pesquisas envolvendo seres humanos. A pesquisa foi aprovada pelo Comitê de Ética em Pesquisa da Associação de Caridade Santa Casa do Rio Grande de Rio Grande-RS, sob o Parecer No 16/2006. Todas as mães entrevistadas assinaram o Termo de Consentimento Livre e Esclarecido.

\section{RESULTADOS E DISCUSSÃO}

A análise bivariada mostrou que o BPN esteve associado positivamente com a idade materna, história de natimortos prévios, BPN prévios e pré-termos prévios, o número de cigarros fumados por dia pela mãe e pelo companheiro, a presença de diabete durante a gestação e a ameaça de aborto na gravidez atual. Além disso, o local de residência $(p=0,067)$ e o tempo de residência na área de exposição $(p=0,058)$ também tendem a associar-se positivamente com o BPN. Associaram-se negativamente com o BPN o peso materno pré-gestacional e altura da mãe, o ganho de peso durante a gestação e o número de consultas pré-natais e, ainda, o aumento do IMC materno $(p=0,062)$ tende a associar-se negativamente com o BPN.

Os resultados obtidos na análise bivariada foram ajustados na análise multivariada e serão apresentados com maiores detalhes a seguir.

\section{Análise multivariada}

A análise multivariada foi realizada baseada no modelo de análise pré-estabelecido. A fim de evitar a exclusão de potenciais fatores de risco e/ou confusão, foi utilizado um nível de significância de 0,20. As Tabelas 1 a 4 apresentam o efeito da exposição aos fatores ambientais ajustados para os fatores de risco e/ou confusão associados com o BPN, as razões de odds bruta e ajustada e seus respectivos intervalos de confiança, bem como os valores do $p$, que referem-se à significância estatística das variáveis ajustadas.

\section{Análise do primeiro nível: características socioeconômi-} cas e demográficas maternas

Inicialmente, foram incluídas na análise, todas as variáveis socioeconômicas e demográficas maternas, independentemente da significância estatística das mesmas na análise bivariada. As variáveis: ausência do companheiro, escolaridade paterna e idade materna foram significativas (Tabela 1) nessa análise $(\mathrm{p}<0,20)$ e foram mantidas para o ajuste do próximo nível, no caso, as variáveis sobre as condições de moradia.

Quanto à ausência de companheiro, embora não tenha mostrado associação quando avaliada na análise bivariada, quando ajustada às demais variáveis do bloco tende a associar-se positivamente com o BPN $(p=0,052)$, ou seja, as mães sem companheiro apresentaram risco maior $(R O=1,64)$ de terem uma criança com $B P N$, em relação às mães com companheiro. No estudo realizado em Pelotas-RS ${ }^{(17)}$, foi encontrada associação positiva entre o BPN e a ausência do companheiro. Da mesma forma, em Campinas$-\mathrm{SP}^{(18)}$, pesquisadores referem associação entre o BPN e as mulheres sem companheiro. O mesmo também foi observado em outro estudo, atribuindo-se esse fato à insegurança psicológica e econômica das mães ${ }^{(19)}$.

O que pode estar contribuindo para que as mães não unidas, ou sem companheiro, apresentem risco aumentado de terem uma criança com BPN, talvez seja a instabilidade emocional pela falta de apoio psicológico do companheiro, a renda familiar reduzida devido a uma única fonte, e os extremos de idade, sobretudo, as mães adolescentes que são as que mais comumente permanecem sem companheiro, e que neste estudo representam $41,1 \%$.

Quanto à escolaridade paterna, o risco da criança nascer com BPN aumentou na análise ajustada quando o pai tinha quatro ou mais anos de escolaridade, comparado com os pais com até três anos de estudo. No entanto, esse risco não foi significativo.

Ainda nesse bloco, na análise bivariada, somente a idade materna mostrou associação positiva com o BPN. Quando se efetuou a análise multivariada, a idade materna mostra tendência a associar-se positivamente com o BPN ( $p=0,052)$, sendo observado um risco maior $(\mathrm{RO}=2,35)$ desse desfecho entre as mães com 35 anos ou mais de idade, quando comparado ao risco observado na análise bivariada $(R O=2,04)$.

Estudos realizados em Campinas ${ }^{(18)}$ e em Guaratinguetá(5), ambos no estado de São Paulo, encontraram associação entre o BPN e a idade materna maior que 35 anos. Nesses estudos, essa mesma associação foi observada em relação às mães adolescentes. Já o estudo realizado em Pelotas-RS ${ }^{(17)}$, não encontrou associação entre o BPN e a idade materna. Dessa maneira, a questão da idade materna como fator de risco para o BPN ainda parece contraditória e não bem esclarecida.

Nesse mesmo bloco, as variáveis que não foram significativas nesse estudo, mas foram em outros estudos, são a escolaridade materna, para a qual foi encontrada associação 
Tabela 1 - Distribuição entre casos e controles do efeito bruto e ajustado das variáveis sobre as características socioeconômicas e demográficas maternas associadas com o BPN. Rio Grande-RS, 2004.

\begin{tabular}{|c|c|c|c|c|c|}
\hline VARIÁVEL & CASOS & CONTROLES & $\begin{array}{c}\text { RAZÃO DE ODDS } \\
\text { BRUTA (IC 95\%) }\end{array}$ & $\begin{array}{l}\text { RAZÃO DE ODDS } \\
\text { AJUSTADA (IC 95\%) }\end{array}$ & $\mathbf{p}$ \\
\hline $\begin{array}{l}\text { Ausência do companheiro }{ }^{\mathrm{a}} \\
\qquad \begin{array}{c}\text { sim } \\
\text { não }\end{array}\end{array}$ & $\begin{aligned} \mathrm{n}=138 & \\
104 & (75,4 \%) \\
34 & (24,6 \%)\end{aligned}$ & $\begin{aligned} \mathrm{n}=409 & \\
319 & (78,0 \%) \\
90 & (22,0 \%)\end{aligned}$ & $\begin{array}{l}1,00 \\
1,15(0,73-1,82)\end{array}$ & $\begin{array}{l}1,00 \\
1,64(0,99-2,71)\end{array}$ & 0,052 \\
\hline $\begin{array}{l}\text { Escolaridade paterna }{ }^{* a} \\
\qquad \begin{array}{c}\leq 3 \\
\geq 4\end{array}\end{array}$ & $\begin{aligned} & \mathrm{n}=134 \\
& 13(9,7 \%) \\
& 121(90,3 \%) \\
&\end{aligned}$ & $\begin{aligned} \mathrm{n}=385 & \\
51 & (13,2 \%) \\
334 & (86,8 \%)\end{aligned}$ & $\begin{array}{l}1,00 \\
1,42(0,74-2,70)\end{array}$ & $\begin{array}{l}1,00 \\
1,62(0,84-3,14)\end{array}$ & 0,15 \\
\hline $\begin{array}{c}\text { Idade materna }^{\mathrm{a}} \\
\begin{array}{c}<20 \\
20-24 \\
25-29 \\
30-34 \\
\geq 35\end{array}\end{array}$ & $\begin{aligned} \mathrm{n}=138 & \\
25 & (18,1 \%) \\
34 & (24,6 \%) \\
29 & (21,0 \%) \\
23 & (16,7 \%) \\
27 & (19,6 \%)\end{aligned}$ & $\begin{aligned} \mathrm{n}=409 & \\
91 & (22,2 \%) \\
134 & (32,8 \%) \\
79 & (19,3 \%) \\
57 & (13,9 \%) \\
48 & (11,7 \%)\end{aligned}$ & $\begin{array}{l}1,00 \\
0,92(0,51-1,65) \\
1,33(0,72-2,46) \\
1,46(0,76-2,83) \\
2,04(1,07-3,90)\end{array}$ & $\begin{array}{l}1,00 \\
0,97(0,53-1,77) \\
1,36(0,71-2,61) \\
1,57(0,79-3,15) \\
2,35(1,19-4,66)\end{array}$ & 0,052 \\
\hline
\end{tabular}

Fonte: Backes, 2004(20)

$a=$ variáveis que permaneceram no modelo de análise $(p<0,20)$.

"Refere-se à escolaridade do pai do recém-nascido, independente da mãe morar ou não com ele.

significativa entre o BPN e as mães com menor escolaridade em Guaratinguetá-SP, sendo a escolaridade acima de oito anos fator de proteção ${ }^{(5)}$. Porém, o mesmo não foi identificado em Pelotas-RS ${ }^{(17)}$. Esse último estudo encontrou associação negativa entre o BPN e a escolaridade materna. Apesar de ter ocorrido diminuição da baixa escolaridade entre as mães em Ribeirão Preto-SP, Sudeste do Brasil, entre 1978-1979 e 1994 houve aumento do BPN(21). Gama et al. ${ }^{(22)}$ observaram em seu estudo que as mulheres com pior nível de instrução eram as que tinham idade entre 20 a 34 anos, com história de gravidez na adolescência.

Em Campinas-SP, houve um aumento do peso ao nascer entre recém-nascidos previdenciários, entre os anos de 1976 a 1980, o que não foi observado entre as categorias com meIhores condições socioeconômicas ${ }^{(18)}$. Da mesma forma, ao ser avaliada a tendência secular do peso ao nascer na cidade de São Paulo-SP, nos anos de 1976 a 1998, foi verificado que a distribuição do peso ao nascer pouco se modificou nesse período, sendo encontrada uma evolução desigual do peso ao nascer de acordo com o nível socioeconômico da população. Evolução favorável foi encontrada nos estratos de nível socioeconômico baixo, provavelmente devido ao melhor desempenho do crescimento intrauterino. Por outro lado, a evolução do peso de nascimento foi desfavorável nos estratos de nível socioeconômico mais alto, o que foi atribuído ao aumento dos nascimentos prematuros ${ }^{(23)}$. Esses estudos poderiam contribuir para explicar a não associação entre as condições socioeconômicas e o BPN observado no presente estudo.

\section{Análise do segundo nível: condições de moradia}

As variáveis relacionadas ao bloco sobre as condições de moradia, do segundo nível, foram adicionadas às variáveis socioeconômicas e demográficas maternas significativas no primeiro nível. Nenhuma das variáveis associou-se com o BPN, nem na análise bruta, nem na análise ajustada. Em
Campinas-SP, observou-se que o desenvolvimento e a meIhoria das condições gerais de vida da população não vem acompanhadas com o aumento do peso ao nascer entre os recém-nascidos, provavelmente devido a outros fatores que estejam impedindo este avanço(18).

\section{Análise do terceiro nível: características biológicas mater-} nas e fatores reprodutivos maternos

Na continuidade, procedeu-se à análise do terceiro nível, com as variáveis sobre as características biológicas maternas e os fatores reprodutivos maternos ajustados para as variáveis socioeconômicas e demográficas maternas significativas, já definidas na análise do primeiro nível. Após esse ajuste, permaneceram no modelo: a altura e o IMC da mãe, o intervalo interpartal e a história de natimortos e BPN prévios (Tabela 2).

A altura materna associou-se negativamente com o BPN na análise bivariada. Na análise multivariada, esta variável igualmente apontou associação negativa em relação ao BPN, ou seja, quanto maior a altura da mãe menor o risco de dar à luz uma criança com baixo peso. A altura de $160 \mathrm{~cm}$ ou mais apresentou efeito protetor também na análise ajustada, e o risco foi ainda menor da criança nascer com $B P N(R O=0,43)$. Este efeito protetor da altura materna sobre o BPN também tem sido constatado por outros autores ${ }^{(17)}$.

$\mathrm{O}$ aumento do IMC apresentou tendência de associação negativa em relação ao BPN na análise bivariada $(p=0,06)$. No entanto, na análise multivariada, o mesmo não foi significativo, contrariamente ao estudo de Santos (1995) ${ }^{(17)}$, onde o aumento do IMC apresentou efeito protetor sobre o BPN. Não é difícil compreender o resultado em nosso estudo, já que para a obtenção deste índice leva-se em consideração o peso materno pré gestacional, o qual também não associou-se significativamente com o desfecho estudado após o ajuste com as demais variáveis. 
Tabela 2 - Distribuição entre casos e controles do efeito bruto das variáveis sobre as características biológicas maternas e fatores reprodutivos associados com o BPN, ajustados para as variáveis socioeconômicas e demográficas maternas significativas. Rio Grande-RS, 2004.

\begin{tabular}{|c|c|c|c|c|c|}
\hline VARIÁVEL & CASOS & CONTROLES & $\begin{array}{c}\text { RAZÃO DE ODDS } \\
\text { BRUTA (IC 95\%) }\end{array}$ & $\begin{array}{l}\text { RAZÃO DE ODDS } \\
\text { AJUSTADA(IC 95\%) }\end{array}$ & $\mathbf{p}$ \\
\hline $\begin{array}{l}\text { Altura }(\mathrm{cm})^{b} \\
\quad<150 \\
150-154 \\
155-159 \\
\geq 160\end{array}$ & $\begin{aligned} \mathrm{n}=138 & \\
10 & (7,2 \%) \\
29 & (21,0 \%) \\
35 & (25,4 \%) \\
64 & (46,4 \%)\end{aligned}$ & $\begin{aligned} \mathrm{n}=409 & \\
18 & (4,4 \%) \\
52 & (12,7 \%) \\
95 & (23,2 \%) \\
244 & (59,7 \%)\end{aligned}$ & $\begin{array}{l}1,00 \\
1,00(0,40-2,46) \\
0,66(0,27-1,57) \\
0,47(0,20-1,07)\end{array}$ & $\begin{array}{l}1,00 \\
1,64(0,45-6,03) \\
1,18(0,34-4,11) \\
0,43(0,13-1,41)\end{array}$ & 0,006 \\
\hline $\begin{array}{c}\text { Índice de massa corporal }\left(\mathrm{IMC}^{* *}\right)^{\mathrm{b}} \\
<18,5 \\
18,5-20 \\
20,01-24,99 \\
325\end{array}$ & $\begin{array}{rr}\mathrm{n}=137 \\
16 & (11,7 \%) \\
19 & (13,9 \%) \\
73 & (53,3 \%) \\
29 & (21,2 \%)\end{array}$ & $\begin{aligned} \mathrm{n}=395 & \\
35 & (8,9 \%) \\
39 & (9,9 \%) \\
212 & (53,7 \%) \\
109 & (27,6 \%)\end{aligned}$ & $\begin{array}{l}1,00 \\
1,06(0,47-2,38) \\
0,75(0,39-1,44) \\
0,58(0,28-1,19)\end{array}$ & $\begin{array}{l}1,00 \\
0,31(0,07-1,43) \\
0,35(0,12-1,06) \\
0,23(0,07-0,74)\end{array}$ & 0,11 \\
\hline $\begin{array}{l}\text { Intervalo interpartal (anos) }{ }^{\mathrm{b}} \\
\qquad \begin{array}{c}<2 \\
2-3 \\
>3\end{array}\end{array}$ & $\begin{array}{rr}\mathrm{n}=78 \\
14 & (17,9 \%) \\
13 & (16,7 \%) \\
51 & (65,4 \%)\end{array}$ & $\begin{aligned} \mathrm{n}=231 & \\
40 & (17,3 \%) \\
54 & (23,4 \%) \\
137 & (59,3 \%)\end{aligned}$ & $\begin{array}{l}1,00 \\
0,68(0,29-1,62) \\
1,06(0,53-2,11)\end{array}$ & $\begin{array}{l}1,00 \\
0,55(0,20-1,51) \\
1,25(0,54-2,91)\end{array}$ & 0,17 \\
\hline $\begin{array}{c}\text { Natimortos prévios }^{b} \\
0 \\
\geq 1\end{array}$ & $\begin{aligned} \mathrm{n}=138 & \\
129 & (93,5 \%) \\
9 & (6,5 \%)\end{aligned}$ & $\begin{aligned} \mathrm{n}=409 & \\
399 & (97,6 \%) \\
10 & (2,4 \%)\end{aligned}$ & $\begin{array}{l}1,00 \\
2,78(1,10-7,00)\end{array}$ & $\begin{array}{l}1,00 \\
3,23(1,03-10,14)\end{array}$ & 0,04 \\
\hline 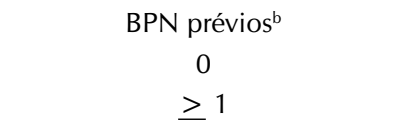 & $\begin{aligned} \mathrm{n}=138 & \\
109 & (79,0 \%) \\
29 & (21,0 \%)\end{aligned}$ & $\begin{aligned} \mathrm{n}=409 & \\
383 & (93,6 \%) \\
26 & (6,4 \%)\end{aligned}$ & $\begin{array}{l}1,00 \\
3,91(2,21-6,93)\end{array}$ & $\begin{array}{l}1,00 \\
5,30(2,54-11,07)\end{array}$ & 0,00 \\
\hline
\end{tabular}

Fonte: Backes, 2004 ${ }^{(20)}$

${ }^{* *} \mathrm{IMC}=$ peso em $\mathrm{kg} /(\text { altura em } \mathrm{m})^{2}$

$b=$ variáveis que permaneceram no modelo de análise, ajustadas para presença do companheiro, escolaridade paterna e idade materna.

O intervalo interpartal também não mostrou associação com o BPN, embora tenha sido observada uma redução no risco de BPN quando este intervalo foi de dois a três anos.

A história de natimortos prévios associou-se positivamente com o BPN tanto na análise bivariada quanto após o ajuste com as demais variáveis na análise multivariada. Quando a mãe teve um ou mais natimortos prévios, o risco aumentado de ter um recém-nascido de BPN observado na análise bruta $(\mathrm{RO}=2,78)$ foi ainda maior na análise ajustada $(\mathrm{RO}=3,23)$. Resultado semelhante foi observado no estudo realizado em Guaratinguetá-SP(5), que encontrou associação significativa entre o BPN e o relato de natimortos prévios. Já no estudo realizado em Pelotas- $\mathrm{RS}^{(17)}$, a história de natimortos prévios não foi significativa para este desfecho.

Da mesma forma como a história de natimortos prévios, a história de BPN prévios também se associou positivamente com o BPN, tanto na análise bruta, como na análise ajustada. Quando a mãe teve um ou mais BPN em gestações anteriores, o risco aumentado de ter uma criança de BPN observado na análise bruta $(R O=3,91)$ também foi maior ainda na análise ajustada $(R O=5,30)$. O mesmo resultado também foi encontrado em outro estudo ${ }^{(17)}$. A história de BPN prévios sugere que, em gestações anteriores, a mãe já estaria exposta a possíveis riscos vividos durante essa última gestação. Nesse estudo, a história de BPN prévios esteve significativamente associada com a idade e altura materna, as quais se constituem em risco para o desfecho estudado.

A história de pré-termos prévios mostrou associação positiva em relação ao BPN na análise bivariada. No entanto, essa associação não foi encontrada na análise multivariada, diferentemente do encontrado em outro estudo ${ }^{(17)}$. Neste estudo, o fato da história de prematuros prévios não ter se mostrado significativa quando ajustada para as variáveis do nível socioeconômico e as características demográficas e biológicas maternas, pode sugerir que algumas dessas variáveis, como a escolaridade e a idade materna, consideradas então fatores de confusão, poderiam estar exercendo algum efeito sobre esta variável. Neste estudo, a história de pré-termos prévios esteve associada significativamente com a escolaridade e a idade materna. Além disso, a altura da mãe também tende a associar-se significativamente com os pré-termos prévios.

Ainda neste bloco, quanto às características biológicas maternas, o peso materno pré-gestacional associou-se negativamente com o BPN na análise bivariada, mas esta associação não foi mais observada após o ajuste das variáveis na análise multivariada. Estudo realizado em Pelotas-RS encontrou efeito protetor sobre o BPN em relação ao peso pré-gestacional, mesmo na análise multivariada ${ }^{(17)}$.

As demais variáveis desse mesmo bloco, que não foram significativas neste estudo foram: o número de gestações anteriores, 
o intervalo interpartal e a história de abortos prévios. Esses resultados foram semelhantes aos encontrados em Pelotas-RS ${ }^{(17)}$. No entanto, em Guaratinguetá/SP(5) foi encontrada associação estatisticamente significativa entre o BPN e mães nulíparas ou com três ou mais filhos, quando comparadas com as mães com um ou dois filhos. Já em Ribeirão Preto/SP, mesmo com a redução do número de multíparas, houve aumento do BPN ${ }^{(21)}$.

\section{Análise do quarto nível: fatores ambientais}

Os fatores ambientais entraram no quarto nível de análise e foram ajustados para as variáveis significativas que permaneceram dos blocos sobre as características socioeconômicas e demográficas maternas, características biológicas maternas e fatores reprodutivos maternos. Desse ajuste, as variáveis que se apresentaram significativas foram o local de residência e o local de trabalho materno (Tabela 3 ).

Nesse bloco referente aos fatores ambientais, o local de residência na área de exposição, embora não tenha apresentado associação significativa, uma vez em que o valor de $p$ encontrado foi um pouco superior ao ponto de corte estabelecido, tanto na análise bruta $(p=0,067)$, como na análise ajustada ( $p=0,057)$, há a possibilidade da existência de uma relação entre exposição ambiental a poluentes e BPN no município do Rio Grande-RS. O risco verificado na análise bruta $(R O=1,87)$ aumentou na análise ajustada $(R O=4,67)$.

Em relação aos estudos que relacionam a poluição ambiental com o BPN, no Brasil, o primeiro realizado nesse sentido ocorreu em São Paulo-SP, em uma pesquisa que envolveu 179 mil nascimentos ocorridos no ano de 1997, mostrando que as mães que ficaram expostas a maiores níveis de poluição do ar no primeiro trimestre da gravidez tiveram recém-nascidos com peso inferior que as demais gestantes da capital paulista ${ }^{(4)}$.

Outro estudo de que se tem conhecimento, também realizado no Brasil, foi feito em Triunfo-RS, o qual apontou uma correlação positiva para BPN e a residência materna próxima ao Pólo Petroquímico. No entanto, essa associação não foi significativa quando outras variáveis foram incluídas na análise de regressão logística condicional, como o fumo, doenças crônicas e a idade materna ${ }^{(2)}$.
Os efeitos da poluição sobre os fetos foram estudados no Estado de São Paulo e foi constatado que os mesmos sofrem com a poluição atmosférica, apesar de estarem protegidos pela placenta e pela estrutura física materna. Segundo este estudo, os abortos e os natimortos foram associados à poluição atmosférica, sendo que a cada oito abortos que ocorreram na capital paulista, diariamente, 1,5 deles podem estar associados à poluição, assim como os natimortos. Nos dias mais poluídos, as mortes fetais a partir do sétimo mês de gestação foram maiores, sendo que, em média, a cada oito óbitos fetais registrados por dia, dois podem estar associados à poluição(24).

No nosso estudo, um dos fatores que pode ter contribuído para o resultado encontrado é o tamanho da amostra proveniente da área exposta, já que, de todos os nascimentos (547), apenas 7,31\% (40) ocorreram na área exposta, dos quais 15 eram casos e 25 eram controles.

Outros estudos tornam-se necessários para avaliar e esclarecer melhor esse efeito sobre o BPN nesta área. Faz-se necessária a realização de estudos que utilizem outros marcadores, além do local de residência, para que possam delimitar com maior precisão quais as áreas mais atingidas ${ }^{(25)}$. Por outro lado, é necessário investigar melhor a exposição das gestantes nos diferentes trimestres da gravidez e, até mesmo, em diferentes estações do ano, envolvendo amostras maiores em relação à área exposta. Todos esses resultados nos fazem refletir também que esses efeitos dos poluentes ambientais, ao menos neste estudo, poderiam estar sendo potencializados pelo tabagismo passivo de outros fumantes no domicílio durante a gestação.

O tempo de residência da mãe na área exposta também tende a associar-se positivamente com o BPN $(p=0,058)$ na análise bivariada, pois quanto mais tempo a mãe morou na área de exposição, maior foi o risco. Já na análise ajustada, esta variável não foi significativa. Nossas análises complementares mostraram que a história de natimortos prévios tende a associar-se significativamente com o tempo de exposição. Entretanto, houve um número muito reduzido de mães com natimortos prévios, apenas três, as quais residiam na área exposta por mais de cinco anos. Das mães que moravam na área de exposição por menos de cinco anos, nenhuma teve natimortos prévios.

Tabela 3 - Distribuição, entre casos e controles, do efeito bruto das variáveis sobre os fatores ambientais associados com o BPN, ajustados para as variáveis socioeconômicas e demográficas maternas, características biológicas e fatores reprodutivos significativos. Rio Grande-RS, 2004.

\begin{tabular}{|c|c|c|c|c|c|}
\hline VARIÁVEL & CASOS & CONTROLES & $\begin{array}{c}\text { RAZÃO DE ODDS } \\
\text { BRUTA (IC 95\%) }\end{array}$ & $\begin{array}{l}\text { RAZÃO DE ODDS } \\
\text { AJUSTADA (IC 95\%) }\end{array}$ & $\mathbf{p}$ \\
\hline $\begin{array}{c}\text { Local de residência } \\
\text { não exposto } \\
\text { exposto }\end{array}$ & $\begin{aligned} \mathrm{n}=138 & \\
123 & (89,1 \%) \\
15 & (10,9 \%)\end{aligned}$ & $\begin{aligned} \mathrm{n}=409 & \\
384 & (93,9 \%) \\
25 & (6,1 \%)\end{aligned}$ & $\begin{array}{l}1,00 \\
1,87(0,95-3,66)\end{array}$ & $\begin{array}{l}1,00 \\
4,67(0,95-22,90)\end{array}$ & 0,057 \\
\hline $\begin{array}{c}\text { Local de trabalho }^{\mathbf{c}} \\
\text { não exposto } \\
\text { exposto }\end{array}$ & $\begin{aligned} \mathrm{n}=138 & \\
124 & (89,9 \%) \\
14 & (10,1 \%)\end{aligned}$ & $\begin{aligned} \mathrm{n}=409 & \\
374 & (91,4 \%) \\
35 & (8,6 \%)\end{aligned}$ & $\begin{array}{l}1,00 \\
1,20(0,62-2,31)\end{array}$ & $\begin{array}{l}1,00 \\
0,23(0,04-1,18)\end{array}$ & 0,08 \\
\hline
\end{tabular}

Fonte: Backes, 2004(20)

$c$ = variáveis que permaneceram no modelo de análise, ajustados para presença do companheiro, escolaridade paterna, idade materna altura e IMC da mãe, intervalo interpartal, história de natimortos prévios e BPN prévios. 
O local de trabalho não apresentou associação com o BPN na análise bivariada. Na análise multivariada, quando ajustado com outras variáveis, o mesmo apontou tendência de associação negativa com o desfecho estudado $(p=0,08)$, apresentando um efeito protetor, ou seja, uma redução do risco $(\mathrm{RO}=0,23)$. No entanto, o número de mães expostas no ambiente de trabalho também foi pequeno, apenas 10,1\% dos casos e 8,6\% dos controles. Ao trabalhar na área exposta, a mãe não sofre os mesmos efeitos da exposição do que quando reside na área exposta. No presente estudo, apenas 29,4\% das mães da amostra exerceram algum tipo de trabalho remunerado. Portanto, o número de mães que trabalhavam na área exposta era pequeno. Além disso, essas mães exerciam atividades de ensino, trabalho administrativo de nível intermediário e inferior, como vendedoras e agentes de venda, auxiliares em serviços públicos e serviço doméstico.
Análise do quinto nível: fatores de risco durante a gestação, intercorrências na gestação e assistência pré-natal e tipo de parto

As variáveis dos quatro níveis analisados até aqui e significativas até o momento, foram conservadas no modelo e utilizadas para ajustar o efeito das variáveis do quinto nível, ou seja, os fatores de risco durante a gestação, as intercorrências na gestação e a assistência pré-natal, e o tipo de parto. Entre essas variáveis, apresentaram-se significativas: o número de cigarros fumados por dia pelo companheiro, a existência de outros fumantes em casa, o consumo de bebida alcoólica pela mãe durante a gestação, o ganho de peso durante a gestação, a ocorrência de hipertensão arterial, diabete, ameaça de aborto durante a gravidez atual e o número de consultas pré-natais (Tabela 4).

No bloco sobre os fatores de risco durante a gestação, a exposição da mãe ao fumo do companheiro, quando avaliada

Tabela 4 - Distribuição, entre casos e controles, do efeito bruto das variáveis sobre os fatores de risco durante a gestação, intercorrências na gestação, assistência pré-natal e tipo de parto, associados com o BPN, ajustados para as características socioeconômicas e demográficas maternas, características biológicas maternas, fatores reprodutivos e fatores ambientais significativos. Rio Grande-RS, 2004.

\begin{tabular}{|c|c|c|c|c|c|}
\hline VARIÁVEL & CASOS & CONTROLES & $\begin{array}{c}\text { RAZÃO DE ODDS } \\
\text { BRUTA (IC 95\%) }\end{array}$ & $\begin{array}{l}\text { RAZÃO DE ODDS } \\
\text { AJUSTADA (IC 95\%) }\end{array}$ & $\mathbf{p}$ \\
\hline $\begin{array}{c}N^{o} \text { cigarros/dia (companheiro) } \\
0-10 \\
11 \mathrm{ou}+\end{array}$ & $\begin{aligned} \mathrm{n}=137 & \\
103 & (75,2 \%) \\
34 & (24,8 \%)\end{aligned}$ & $\begin{aligned} \mathrm{n}=409 & \\
342 & (83,6 \%) \\
67 & (16,4 \%)\end{aligned}$ & $\begin{array}{l}1,00 \\
1,68(1,05-2,69)\end{array}$ & $\begin{array}{l}1,00 \\
2,27(0,87-5,91)\end{array}$ & 0,09 \\
\hline $\begin{array}{l}\text { Outros fumantes em casa }^{d} \\
\text { não } \\
\text { sim }\end{array}$ & $\begin{array}{rr}\mathrm{n}=138 \\
108 & (78,3 \%) \\
30 & (21,7 \%)\end{array}$ & $\begin{aligned} \mathrm{n}=409 & \\
319 & (78,0 \%) \\
90 & (22,0 \%)\end{aligned}$ & $\begin{array}{l}1,00 \\
0,98(0,61-1,57)\end{array}$ & $\begin{array}{l}1,00 \\
0,38(0,10-1,45)\end{array}$ & 0,16 \\
\hline $\begin{array}{l}\text { Consumo de bebidas alcoólicas }{ }^{\mathrm{d}} \\
\qquad \begin{array}{c}\text { não } \\
\text { sim }\end{array}\end{array}$ & $\begin{array}{rr}\mathrm{n}=138 \\
80 & (58,0 \%) \\
58 & (42,0 \%)\end{array}$ & $\begin{aligned} \mathrm{n}= & 409 \\
257 & (62,8 \%) \\
152 & (37,2 \%)\end{aligned}$ & $\begin{array}{l}1,00 \\
1,22(0,82-1,81)\end{array}$ & $\begin{array}{l}1,00 \\
2,10(0,94-4,71)\end{array}$ & 0,07 \\
\hline $\begin{array}{c}\text { Ganho de peso }(\mathrm{kg})^{\mathrm{d}} \\
\quad<8 \\
8-12 \\
>12\end{array}$ & $\begin{array}{rr}\mathrm{n}=128 \\
59 & (46,1 \%) \\
40 & (31,3 \%) \\
29 & (22,7 \%)\end{array}$ & $\begin{aligned} \mathrm{n}=378 & \\
72 & (19,0 \%) \\
119 & (31,5 \%) \\
187 & (49,5 \%)\end{aligned}$ & $\begin{array}{l}1,00 \\
0,41(0,24-0,67) \\
0,18(0,11-0,31)\end{array}$ & $\begin{array}{l}1,00 \\
0,39(0,16-0,95) \\
0,08(0,02-0,29)\end{array}$ & 0,0003 \\
\hline $\begin{array}{r}\text { Hipertensão arterial }^{\mathrm{d}} \\
\text { não } \\
\operatorname{sim}\end{array}$ & $\begin{array}{rr}\mathrm{n}=138 \\
102 & (73,9 \%) \\
36 & (26,1 \%)\end{array}$ & $\begin{aligned} \mathrm{n}=407 & \\
325 & (79,9 \%) \\
82 & (20,1 \%)\end{aligned}$ & $\begin{array}{l}1,00 \\
1,39(0,89-2,19)\end{array}$ & $\begin{array}{l}1,00 \\
3,77(1,40-10,17)\end{array}$ & 0,008 \\
\hline Diabete $^{d}$ & $\begin{aligned} \mathrm{n}=135 & \\
122 & (90,4 \%) \\
13 & (9,6 \%)\end{aligned}$ & $\begin{aligned} \mathrm{n}=404 & \\
387 & (95,8 \%) \\
17 & (4,2 \%)\end{aligned}$ & $\begin{array}{l}1,00 \\
2,42(1,14-5,13)\end{array}$ & $\begin{array}{l}1,00 \\
4,65(0,82-26,40)\end{array}$ & 0,08 \\
\hline $\begin{array}{r}\text { Ameaça de aborto }^{d} \\
\text { não } \\
\text { sim }\end{array}$ & $\begin{aligned} \mathrm{n}=138 & \\
125 & (90,6 \%) \\
13 & (9,4 \%)\end{aligned}$ & $\begin{aligned} \mathrm{n}=409 & \\
392 & (95,8 \%) \\
17 & (4,2 \%)\end{aligned}$ & $\begin{array}{l}1,00 \\
2,39(1,13-5,07)\end{array}$ & $\begin{array}{l}1,00 \\
6,27(1,53-25,76)\end{array}$ & 0,01 \\
\hline $\begin{array}{l}\text { Número de consultas pré-natais }{ }^{\mathrm{d}} \\
\qquad \begin{array}{c}0 \\
<5 \\
\geq 5\end{array}\end{array}$ & $\begin{array}{rr}\mathrm{n}=138 \\
17 & (12,3 \%) \\
50 & (36,2 \%) \\
71 & (51,4 \%)\end{array}$ & $\begin{aligned} \mathrm{n}=409 & \\
26 & (6,4 \%) \\
98 & (24,0 \%) \\
285 & (69,7 \%)\end{aligned}$ & $\begin{array}{l}1,00 \\
0,78(0,38-1,57) \\
0,38(0,19-0,74)\end{array}$ & $\begin{array}{l}1,00 \\
0,32(0,07-1,48) \\
0,17(0,04-0,78)\end{array}$ & 0,049 \\
\hline
\end{tabular}

Fonte: Backes, 2004(20)

$d$ = variáveis que permaneceram no modelo de análise, ajustadas para presença do companheiro, escolaridade paterna, idade materna, altura, IMC, intervalo interpartal, natimortos prévios, BPN prévios, local de residência e local de trabalho. 
de forma dicotômica, também não mostrou associação com o BPN em nenhum momento das análises efetuadas. No entanto, embora o fato do companheiro ter fumado mais de 10 cigarros por dia tenha sido significativo em relação ao BPN na análise bruta, na análise ajustada, apesar do risco ser ainda mais elevado ( $R O=2,27$ ), os resultados não demonstraram associação significativa com o BPN $(p=0,09)$. Quando o número de cigarros fumados por dia era 11 ou mais, os companheiros fumaram cerca de duas vezes mais do que as mães, tanto entre os casos como entre os controles. Outro estudo realizado em Rio Grande-RS também verificou uma alta prevalência do hábito de fumar dos pais (42\%) no município, inclusive maior do que a prevalência observada para as mães neste município ${ }^{(26)}$.

O fato de a mãe fumar ou não, não foi significativo em relação ao BPN, nem na análise bruta e nem na análise ajustada. Entretanto, ao se avaliar o número de cigarros fumados por dia pela mãe, encontrou-se associação positiva com o BPN na análise bivariada. Na análise multivariada, essa associação desapareceu. Esse resultado é diferente do apresentado no estudo de Pelotas-RS ${ }^{(17)}$, o qual encontrou associação positiva entre o BPN e o tabagismo no terceiro trimestre da gestação.

Em nosso estudo, uma maior proporção de fumantes foi encontrada entre as mães de renda mais baixa e de menor grau de instrução. Em Ribeirão Preto-SP, mesmo com a redução do número de mães fumantes, foi observado um aumento do BPN ${ }^{(21)}$.

Outras variáveis deste mesmo bloco, como: exposição da mãe a outros fumantes em casa, a exposição materna ao fumo no ambiente de trabalho e o trabalho materno remunerado, não foram significativas na análise bruta e nem na análise ajustada.

O trabalho materno fora de casa durante a gestação, embora não tenha sido significativo, aumentou o risco de a mãe ter um recém-nascido com BPN, contrário ao estudo de Santos ${ }^{(17)}$ que, embora também não tenha encontrado associação significativa com o BPN, encontrou um risco menor da criança nascer com BPN quando a mãe trabalhou fora de casa. Estudo realizado em Campinas-SP ${ }^{(18)}$ constatou que o trabalho materno fora de casa não exerceu efeito sobre o peso ao nascer, o que também já tem sido relatado por Passini Jr. ${ }^{(19)}$, que considera esta variável como um possível fator de risco, somente nos casos em que a jornada de trabalho exige muito esforço físico e muitas horas na posição em pé.

Do total da amostra, $61 \%$ das mães eram do lar, 9,5\% eram estudantes e, apenas $29,4 \%$ exerceram algum tipo de trabalho remunerado, sendo este do tipo manual não qualificado ou semi-especializado. Resultados semelhantes já haviam sido encontrados sobre o trabalho remunerado das mães deste município, com uma pequena diferença em relação ao número de mães que trabalharam fora, ou seja, a prevalência era um pouco maior $(38 \%)^{(6)}$.

O consumo de bebida alcoólica por parte da mãe durante a gestação não se associou com o BPN na análise bivariada. Quando analisado junto com outras variáveis, na análise multivariada, foi encontrada tendência de associação positiva com o BPN $(p=0,07)$. Estudo realizado em Pelotas-RS não encontrou efeito negativo entre este consumo por parte da mãe na gestação e o BPN ${ }^{(17)}$. Em nossa pesquisa houve maior consumo desta bebida entre as mulheres com melhor renda, o que também já havia sido constatado em Pelotas-RS ${ }^{(27)}$.

Apesar de ser contraindicado o consumo de bebidas alcoólicas durante a gestação, este hábito foi bastante frequente neste estudo ( $42 \%$ dos casos e 37,2\% dos controles). Têm sido descritas as consequências deste uso para o concepto, como o retardo do crescimento intrauterino, retardo mental, redução do peso ao nascer e possível aparecimento de malformações ${ }^{(27)}$.

Em relação ao bloco de variáveis sobre o ganho de peso e as intercorrências durante a gestação, o ganho de peso materno durante a gestação associou-se negativamente com o BPN nos dois tipos de análise. Desta maneira, o ganho de peso exerceu um efeito protetor sobre o BPN. Quanto maior o ganho de peso durante a gestação, menor o risco de a criança nascer com BPN. Quando a mãe aumentou mais de $12 \mathrm{~kg}$, o risco de ter um recém-nascido de BPN foi ainda mais reduzido na análise ajustada $(R O=0,08)$, quando comparado à análise bruta $(R O=0,18)$. Estudo realizado em Pelotas- $R S^{(17)}$ também encontrou o mesmo resultado. Um estudo de coorte retrospectiva, realizado em São Paulo ${ }^{(28)}$, verificou que o BPN esteve correlacionado com o ganho de peso materno menor ou igual a $10 \mathrm{~kg}$ durante a gestação. $\mathrm{O}$ peso insuficiente (2500g a 2999g) foi mais comum quando o ganho de peso foi menor ou igual a $12 \mathrm{~kg}$. Para a criança nascer com peso normal (3000g ou mais), foi necessário que a mãe aumentasse 12 kg na gestação. Já o aumento acima de 16 kg na gestação não esteve associado com o aumento do peso do recém-nascido. Para esses autores, a nutrição materna e o peso do recém-nascido apresentam uma proporcionalidade direta.

Neste estudo, das mães que tiveram recém-nascidos com BPN, 46,1\% delas aumentaram menos de oito quilos, contra apenas $15,4 \%$ das mães que tiveram um recém-nascido de peso normal. Opostamente, 53,5\% das mães que tiveram crianças com peso normal, aumentaram mais de 12 quilos na gestação, contra apenas $22,7 \%$ das mães que tiveram crianças com BPN. Todos esses dados confirmam o que já foi apontado por outros autores quanto à importância do ganho de peso adequado durante a gestação para evitar o nascimento de crianças com baixo peso.

A ocorrência de hipertensão arterial durante a gestação, considerando como hipertensão arterial a pressão arterial $\geq$ 140/90 mmHg, não foi significativa na análise bruta. Entretanto, ao entrar no modelo de análise multivariada, a mesma apresentou associação positiva com o BPN. Santos ${ }^{(17)}$ também encontrou associação positiva entre o BPN e a história de hi-

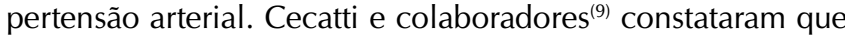
há uma maior ocorrência de hipertensão arterial em mães negras durante a gestação, este fato não foi observado no presente estudo. Assim como não houve associação com a idade materna. No estudo de coorte realizado em seis capitais brasileiras $^{(29)}$, os distúrbios hipertensivos foram mais frequentes entre as mulheres obesas. Alta prevalência de hipertensão transitória e crônica durante a gestação foi encontrada em Pelotas-RS ${ }^{(27)}$. A hipertensão arterial transitória ou crônica pode 
levar ao retardo do crescimento intrauterino, prematuridade e alta mortalidade perinatal ${ }^{(30)}$ o que poderia explicar a sua associação com o BPN encontrada no presente estudo.

Estudo retrospectivo realizado em 2002, num hospital público de São Paulo, avaliou 604 prontuários de gestantes internadas e identificou 22 casos (3,64\%) de Doença Hipertensiva Específica da Gestação. Destas, 40,9\% eram primigestas e $45,45 \%$ eram adolescentes ${ }^{(31)}$.

A ocorrência de diabete durante a gestação associou-se positivamente com o BPN na análise bivariada. Na análise multivariada, a mesma apenas manteve uma tendência de associação positiva com o BPN $(p=0,08)$. Em Pelotas-RS ${ }^{(17)}$ foi encontrada associação negativa entre o BPN e a história de diabete. Constatou-se que as mães que apresentaram um risco aumentado para a diabete gestacional eram obesas a apresentaram menor risco para microssomia ${ }^{(29)}$. Embora o esperado para os filhos de mães diabéticas é que os mesmos sejam macrossômicos, este estudo mostra que as mães que apresentaram diabete durante a gestação possuem tendência a ter recém-nascidos com BPN, o que pensamos poderia estar associado à presença de hipertensão arterial, decorrente da obesidade. Por outro lado, é importante destacar também o número pequeno de mães diabéticas na amostra.

A infecção urinária, ainda pertencente a este bloco, não apresentou associação com o BPN na análise bruta e nem na análise ajustada. No entanto, em Pelotas-RS ${ }^{(17)}$ foi encontrada associação positiva entre o BPN e a infecção do trato urinário. Neste estudo, a infecção urinária associou-se significativamente com a renda familiar.

A ameaça de aborto associou-se significativamente com o BPN, apresentando um risco aumentado na análise bruta $(R O=2,39)$ e, um risco ainda maior na análise ajustada $(\mathrm{RO}=6,27)$. Embora não se tenha encontrado estudos disponíveis na literatura que associem esta variável com o BPN, sabemos que as mães que apresentam ameaça de aborto durante a gestação, possuem uma probabilidade maior de ter recém-nascidos prematuros e com BPN, devido a uma série de intercorrências que podem acometê-las, tais como, placenta prévia, bolsa rota, trabalho de parto prematuro, entre outras.

No bloco sobre a assistência pré-natal e o tipo de parto, o número de consultas pré-natais associou-se negativamente com o BPN na análise bivariada, bem como na análise multivariada. Quando o número de consultas pré-natais foi maior ou igual a cinco, o risco reduziu na análise bruta $(\mathrm{RO}=0,38)$ e, na análise ajustada essa redução do risco foi ainda maior $(R O=0,17)$. Quanto maior o número de consultas pré-natais, menor o risco de a criança nascer com BPN, como também já foi constatado por outros autores ${ }^{(5,14)}$. Apenas cerca da metade $(51,5 \%)$ das mães de toda a amostra iniciaram o pré-natal no primeiro trimestre e, 7,9\% (43) das mães não realizaram nenhuma consulta pré-natal.

Foi encontrada associação negativa entre o número de consultas pré-natais e o BPN na cidade de Pelotas-RS ${ }^{(17)}$. Outro estudo realizado em Pelotas- $\mathrm{RS}^{(32)}$ sobre a atenção pré-natal, constatou uma incidência de BPN 2,5 vezes maior entre as mães que não realizaram pré-natal, quando comparadas com aquelas que realizaram cinco ou mais consultas, sendo que a falta de pré-natal foi mais frequente entre as mães mais pobres, especialmente entre as adolescentes ou acima de 40 anos de idade. No estado de São Paulo foi verificado que o aumento do número de consultas pré-natais reduz a prevalência de BPN e/ou prematuridade, sendo que quando o número de consultas era de zero a três, a prevalência do BPN e/ou da prematuridade era $14 \%$, caindo para $4 \%$ quando o número de consultas era 07 (sete) ou mais ${ }^{(14)}$.

Portanto, os resultados encontrados nesse estudo vêm somar-se aos dos demais autores ${ }^{(5,14,33)}$, ratificando a importância da assistência pré-natal para evitar desfechos gestacionais desfavoráveis, sobretudo o BPN. Ressalte-se a importância de que esse pré-natal seja iniciado o mais precocemente possível, tenha a periodicidade correta e possa ser oferecido com qualidade à todas as gestantes.

Estudo qualitativo realizado no interior do estado de Goiás, com onze gestantes, constatou que as mesmas apresentaram conhecimento deficiente em relação à gestação, déficit de autocuidado e alguns diagnósticos de risco. As autoras consideram que os padrões mínimos preconizados frente à política de atendimento à gestante durante o pré-natal precisam ser repensados e reestruturados, pois a mesma deixa a desejar e enfatiza a consulta médica individual, desconsiderando a assistência que pode ser oferecida pelos demais profissionais, como no caso do enfermeiro, que também está habilitado para realizar consultas individuais de enfermagem, além de atendimento grupal interdisciplinar, e alegam que acompanhar as modificações que ocorrem durante a gestação e os reais problemas, requer, na maioria das vezes, "a aproximação do enfermeiro com a pessoa da gestante em dimensões mais subjetivas aumentando a interação, desenvolvendo a confiança, aumentando a credibilidade da enfermagem e gerando bases para a assistência mais humanizada e de melhor qualidade"(34).

Neste estudo o tipo de parto não se associou com o BPN nos dois tipos de análise efetuadas. Estudo realizado em Guaratinguetá/SP, apesar de ter encontrado uma taxa maior de cesáreas $(70 \%)$, detectou associação positiva entre o parto normal e o BPN ${ }^{(5)}$. No estudo de coorte de base populacional realizado em Ribeirão Preto/SP também foi constatado um aumento na prevalência de BPN entre os nascidos de parto vaginal e esse fato foi atribuído especialmente ao crescimento do número de recém-nascidos prematuros e de mães sem companheiro ${ }^{(35)}$. Estudo realizado em São José do Rio Preto-SP, no ano de 1992, observou que as cesáreas têm aumentado com a paridade e estiveram associadas significativamente com a idade materna e a realização da laqueadura, sendo mais freqüentes entre os estratos sociais mais elevados ${ }^{(36)}$.

Com relação a Rio Grande-RS, pesquisadores ${ }^{(6)}$ referem que há uma prevalência muito alta $(43 \%)$ de cesarianas no município. No presente estudo, quando considerada a amostra como um todo, 53\% das mães realizaram parto cesáreo. Esse índice ainda mais elevado poderia ser explicado pelo tipo de estudo caso-controle, o qual dirige sua atenção para recém-nascidos de baixo peso, já determinando assim um desfecho gestacional desfavorável, o que poderia estar associado a um número maior de cesarianas. É importante também remarcar que neste estudo, das crianças nascidas de cesarianas (53\%), 60,2\% aconteceram 
no Hospital Universitário e 42,2\% na Santa Casa. O Hospital Universitário tem sido considerado para gestação de risco por possuir uma unidade de cuidados intensivos neonatais.

O BPN é um indicador complexo, conseqüência de vários fatores de risco e/ou confusão envolvidos, que interagem isolada ou simultaneamente, em maior ou menor intensidade, de acordo com a exposição e as condições oferecidas pelo ambiente em que vive a gestante. Os estudos disponíveis na literatura não são unânimes ao mostrar estes fatores e, os mesmos apresentam diferenças, dependendo do tipo de estudo e do local onde foram realizados.

Os profissionais da área da saúde estão despertando aos poucos para a consciência de que a poluição ambiental gerada pelos processos industriais interfere no processo gestacional das mães expostas, ocasionando BPN, provocando malformações e, inclusive, causando a morte de embriões e fetos.

Como profissionais da saúde e de enfermagem, deve-se considerar o BPN como um indicador das condições de saúde das gestantes e, em decorrência de todas as implicações e desdobramentos que esta condição pode determinar, considerá-lo, em conseqüência, um indicador das condições desfavoráveis vivenciadas pela mãe antes e durante a gestação.

\section{CONSIDERAÇÕES FINAIS}

Após a análise multivariada, foram identificados como fatores de risco para o BPN os natimortos prévios, BPN prévios, a presença de hipertensão arterial durante a gestação e a ameaça de aborto durante a gravidez atual. Além disso, também apresentam tendência de associar-se positivamente com o BPN a ausência do companheiro $(p=0,052)$, o aumento da idade materna $(p=0,052)$, o local de residência $(p=0,057)$, o número de cigarros fumados por dia pelo companheiro $(p=0,09)$, o consumo de bebidas alcoólicas $(p=0,07)$ e o diabete $(p=0,08)$. Foram identificados como fatores de proteção para o BPN o aumento da altura materna, o ganho de peso e o aumento do número de consultas pré-natais.

Nossos resultados mostram também a possibilidade da existência de uma relação entre exposição ambiental a poluentes e BPN no município do Rio Grande-RS, embora não tenha sido encontrada uma associação significativa, uma vez em que o valor de $p$ encontrado foi um pouco superior ao ponto de corte estabelecido. Tornam-se necessários nesse município, outros estudos para avaliar e esclarecer melhor o efeito da poluição ambiental sobre o BPN, através da utilização de outras metodologias, como por exemplo, o uso de biomarcadores e de marcadores locais da poluição, nos diferentes trimestres da gravidez e, até mesmo, em diferentes estações do ano, envolvendo amostras maiores em relação à área exposta.

Acreditamos que, com essa pesquisa, foi possível identificar os principais fatores de risco a que estão expostas as gestantes e que podem determinar a ocorrência do BPN de seus conceptos, aos quais vêm somar-se àqueles, decorrentes de uma maior exposição a poluentes, devido ao fato de residirem nas proximidades das indústrias. Nossos resultados permitem o aporte de subsídios para a prevenção de danos à saúde, decorrentes da exposição a poluentes ambientais, assim como, podem contribuir para o planejamento e implementação de políticas públicas que visem o desenvolvimento sustentável, mantendo a saúde e a qualidade de vida da população, uma vez em que é indispensável e mais eficaz prevenir os problemas de saúde e a degradação ambiental, do que corrigir os danos já ocorridos.

\section{REFERÊNCIAS}

1. Leite JC, Schüler-Faccini L. Defeitos congênitos em uma região de mineração de carvão. Rev Saúde Pública. 2001; 35(2): 136-141.

2. Oliveira LM, Stein N, Sanseverino MT, Vargas VM, Fachel JM, Schüler L. Reproductive outcomes in an area adjacent to a petrochemical plant in southern Brazil. Rev. Saúde Públ. 2002; 36: 81-7.

3. Franco T, Druck G. Padrões de industrialização, riscos e meio ambiente. Ciênc. saúde colet. 1998; 3(2): 61-72.

4. Gouveia N, Bremner SA, Novaes HM. Association between ambient air pollution and birth weight in São Paulo, Brazil. J Epidemiol Comunity Health. 2004; 58: 11-17.

5. Nascimento LFC, Gotlieb SLD. Fatores de risco para o baixo peso ao nascer, com base em informações da declaração de nascido vivo em Guaratinguetá, SP, no ano de 1998. Inf. Epidemiol. 2001; 10(3): 113-20.

6. César JÁ, Horta BL. Desigualdade e perversidade: epidemiologia do adoecer no extremo-sul do Brasil. Rio Grande: Ed. da Fundação Universidade do Rio Grande; 1997.

7. Victora CG. Factores de riesgo em las IRA bajas. In: Benguigui Y, Lópes-Antuñano FJ, Yunes J. Infecciones respiratorias en niños. Washington: OPAS; 1996. p. 45-63.

8. Alberman E. Are our babies becoming bigger? J R Soc Med. 1991 may.; 84(5): 257-260.

9. Cecatti JG, Machado MR, Santos FF, Marussi EF. Curva dos valores normais de peso fetal estimado por ultra-sonografia, segundo a idade gestacional. Cad. Saúde Pública. $2000 ; 16(4)$ : 1083-90.

10. Silva AAM, Lamy-Filho F, Alves MTSSB, Coimbra LC, Bettiol $\mathrm{H}$, Barbieri MA. Risk factors for low birthweight in north-east Brazil: the role of cesarean section. Paediatr Perinat Epidemiol. 2001; 15: 257-74.

11. Lekea-Karanika V, Tzoumaka-Bakoula C, Matsaniotis NS. Sociodemographic determinants of low birth weight in Greece: a population study. Paediatr Perinat Epidemiol. 1999 jan.; 13(1): 65-77.

12. Haidar FH, Oliveira UF, Nascimento LF. Escolaridade materna: correlação com os indicadores obstétricos. Cad. Saúde Pública. 2001; 17(4): 1025-9.

13. Bicalho CG, Barros Filho AA. Peso ao nascer e influência do consumo de cafeína. Rev. Saúde Públ. 2002; 36(2): 180-7. 
14. Kilsztajn S, Rossbach A, Carmo MSN, Sugahara GTL. Assistência pré-natal, baixo peso e prematuridade no Estado de São Paulo, 2000. Rev. Saúde Públ. 2003; 37(3): 303-10.

15. Backes MTS. O perfil de nascimentos no município de Rio Grande-RS no período de janeiro a junho de 2003. Anais do XII Congresso de Iniciação Científica; 2003 nov 5; Pelotas (RS), Brasil. Pelotas: UCPel; 2003. p. 283.

16. Brasil. Ministério da Saúde. Resolução $n^{\circ}$. 196. Diretrizes e normas técnicas regulamentadoras de pesquisa envolvendo seres humanos. Brasília (DF); 1996.

17. Santos IS. Consumo de cafeína e baixo peso ao nascer: um estudo de casos e controles de base populacional. [Tese]. Porto Alegre (RS): Faculdade de Medicina, Universidade Federal do Rio Grande do Sul; 1995. 252 f. Doutorado em Clínica Médica.

18. Mariotoni CG, Barros Filho AA. Peso ao nascer e características maternas ao longo de 25 anos na maternidade de Campinas. J. Pediatr. (Rio J.). 2000 jan.-fev.; 76(1): 55-64.

19. Passini Júnior R. Associação entre trabalho materno e resultados gestacionais. [Tese]. Campinas (SP): Universidade Estadual de Campinas; 1996. 168 f. Doutorado em Ciências Médicas.

20. Backes MTS. O baixo peso ao nascer em recém-nascidos de mães residentes nas comunidades próximas ao parque industrial do município de Rio Grande-RS: um estudo de casos e controles. [Dissertação]. Rio Grande (RS): Fundação Universidade Federal do Rio Grande (FURG); 2004. Mestrado em Enfermagem.

21. Goldani MZ, Bettiol H, Barbieri MA. Maternal age, social changes, and pregnancy outcome in Ribeirão Preto, Southeast Brazil, in 1978-79 and 1994. Cad. Saúde Pública. 2000 out.-dez.; 16(4): 1041-7.

22. Gama SGN, Szwarcwald CL, Leal MC. Experiência de gravidez na adolescência, fatores associados e resultados perinatais entre puérperas de baixa renda. Cad. Saúde Pública. 2002; 18(1): 153-61.

23. Monteiro CA, Benício MH, Ortiz LP. Tendência secular do peso ao nascer na cidade de São Paulo (1976-1998). Rev. Saúde Públ. 2000; 34(6): 26-40.

24. Pereira LA, Loomis D, Conceição GM, Braga AL, Arcas RM, Kishi HS, Singer JM, Böhm GM, Saldiva PH. Association between air pollution and intrauterine mortality in São Paulo, Brazil. Environ Health Perspect. 1998; 106: 325-29.

25. Huang $Y L$, Batterman S. Selection and evaluation of air pollution exposure indicators based on geographic areas. Science Total Environment. 2000; 253(1-3): 127-44.

26. Prietsch SO. Doença respiratória aguda baixa em menores de cinco anos: fatores de risco e prevalência em Rio Grande, RS. [Dissertação]. Porto Alegre (RS): Universidade Federal do Rio Grande do Sul; 1999. Mestrado em Medicina.

27. Garcias GL. Diagnóstico comunitário e análise do discurso a respeito da compreensão popular em relação à causalidade dos defeitos congênitos. [Tese]. Porto Alegre (RS): Universidade Federal do Rio Grande do Sul; 1999. 171 f. Doutorado em Genética e Biologia Molecular.

28. Lizo CLP, Azevedo-Liso Z, Aronson E, Segre CAM. Relação entre ganho de peso materno e peso do recém-nascido. J. Pediatr. (Rio J.). 1998; 74(2): 114-8.

29. Nucci LB, Duncan BB, Mengue SS, Branchtein L, Schmidt $\mathrm{MI}$, Fleck ET. Assessment of weight gain during pregnancy in general prenatal care services in Brazil. Cad. Saúde Pública. 2001 nov.-dez.; 17(6): 1367-74.

30. Cunninghan FG, Lindheimer MD. Hypertension in pregnancy. N Engl J Med. 1992; 326(14): 927-32.

31. Gonçalves, R, Fernandes RAQ, Sobral DH. Prevalência da doença hipertensiva específica da gestação em hospital público de São Paulo. Rev Bras Enferm. 2005; 58(1): 61-4.

32. Halpern R, Barros FC, Victora CG, Tomasi E. Atenção pré-natal em Pelotas, Rio Grande do Sul, Brasil, 1993. Cad. Saúde Pública. 1998.; 14(3): 487-92.

33. Carniel EF, Zanolli ML, Antônio MARGM, Morcillo AM. Determinantes do baixo peso ao nascer a partir das declarações de nascidos vivos. Rev. bras. epidemiol. 2008; 11(1): 169-79.

34. Pereira SVM, Bachion MM. Diagnósticos de Enfermagem identificados em gestantes durante o pré-natal. Rev Bras Enferm. 2005 nov.-dez.; 58(6): 659-664.

35. Barbieri MA, Silva AAM, Bettiol H, Gomes UA. Risk factors for the increasing trend in low birth weight among live births born by vaginal delivery, Brazil. Rev. Saúde Públ. 2000; 34(6): 596-602.

36. Moraes MS, Goldenberg P. Cesáreas: um perfil epidêmico. Cad. Saúde Pública. 2001; 17(3): 509-19. 